\title{
LA GEOGRAFÍA DE LA POBLACIÓN EN LA PLANIFICACIÓN DEL TERRITORIO
}

\author{
Vicente Gozálvez Pérez \\ Universidad de Alicante
}

RESUMEN

Se ofrecen datos sobre la dedicación de los geógrafos españoles a los estudios de población, y sobre la demanda actual de informes geodemográficos en trabajos de planificación. Se señalan algunos ejemplos que muestran la utilidad práctica de los estudios de población, tanto a nivel nacional como en aspectos sectoriales: en planificación rural y para los espacios urbanos en sus diversas escalas de actuación (áreas urbanas o metropolitanas, ciudad o espacios intraurbanos). En el último apartado se hacen algunas indicaciones sobre la insuficiencia de las fuentes geodemográficas disponibles, sobre todo para la planificación a nivel municipal e intramunicipal.

\section{RÉSUMÉ}

On offre des renseignements sur la dédicace des géographes espagnols aux études de la population, et sur la demande actuelle de rapports géodemographiques dans des travaux de planification. On donne quelques exemples qui montrent I'utilité pratique des études de la population, tant à niveau national qu'en aspects sectoriels: en planification rurale et pour les espaces urbaines et métropolitaines, ville ou espaces intraurbanos. Dans le dernier paragraphe quelques indications sont faites sur l'insuficence des sources géodémographiques disponibles, sur tout pour la planification à niveau municipal et intramunicipal.

\section{Nota sobre docencia e investigación}

Es un hecho reconocido que los estudios de Geografía de la población se han incrementado de forma muy notable durante el último cuarto de siglo. Así, en una publicación de 1984, J.I. Clarke, Presidente de la Comisión de Geografía de la Población de la U.G.I. señala que 
esta temática supone el $10 \%$ de las publicaciones geográficas ${ }^{1}$. A nivel de España, este porcentaje probablemente es muy similar; así, si se contabilizan, como muestra, las tesis doctorales y memorias de licenciatura de las Universidades Complutense de Madrid, de Barcelona y de Valencia, se obtienen las siguientes cifras: de un total de 176 tesis doctorales de Geografía, sólo 8 o el 5\% son de geografía de la población, pero de 266 memorias de licenciatura se dedican a esta materia el $9 \%$ o 24 en cifras absolutas ${ }^{2}$. Sin embargo es muy probable que estas investigaciones aumenten en España, a juzgar por otros indicadores.

Uno de ellos puede ser las especialidades que declaran cultivar los aspirantes a socios de la Asociación de Geógrafos Españoles. Estos han de rellenar un cuestionario donde, entre otros datos, se les pide esta información, aunque estimo que las declaraciones no pueden alcanzar una fiabilidad absoluta por la forma limitada que el impreso ofrece de las distintas materias o especialidades geográficas. Para obviar este inconveniente ahora se recogen las respuestas sólo a seis especialidades que por su tradición pueden gozar de mayor grado de aceptación y sin posibilidades de confusión sobre sus contenidos:

Período de observación: septiembre 1983 a mayo 1987; número de inscripciones 268; número de impresos con respuestas en la pregunta «especialidades que cultiva», 248.

\begin{tabular}{|c|c|c|c|c|c|}
\hline ESPECIALIDAD & TOTAL & $\%$ & $\begin{array}{c}\text { ENTRE LOS DE } \\
\text { LA ESPECIALIDAD, } \\
\text { \% QUE LA CULTIVAN } \\
\text { COMO MATERIA } \\
\text { UNICA }\end{array}$ & $\begin{array}{l}\text { COMPARTIDA } \\
\text { CON OTRA }\end{array}$ & $\begin{array}{l}\text { \% DE ESPE- } \\
\text { CIALISTAS } \\
\text { SOBRE EL } \\
\text { TOTAL }\end{array}$ \\
\hline Geomorfología & 49 & 19,8 & 18,4 & 28,6 & 3,6 \\
\hline Climatología & 50 & 20,2 & 16,0 & 24,0 & 3,2 \\
\hline Biogeografía & 33 & 13,3 & 0,0 & 21,2 & 0,0 \\
\hline Geografía población & 81 & 32,7 & 12,3 & 28,4 & 4,0 \\
\hline Geografía urbana & 95 & 38,3 & 15,8 & 31,6 & 6,0 \\
\hline Geografía agraria & 79 & 31,8 & 20,2 & 29,1 & 6,4 \\
\hline
\end{tabular}

Fuente: Secretaría de la AGE, elaboración propia.

1 CLARKE, J. L, “Geography and Population. Approaches and Applications”, Oxford, Pergamon, 1984. Traducido parcialmente en Est. Geográficos, 178-179. Madrid, 1985, p. 7.

2 Las cifras por Universidades son las siguientes: Universidad Complutense de Madrid:

Período 1934-81: 109 Tesis doctorales, de las que 5 son de geografía de la población.

Período 1976-80: 58 Memorias de Licenciatura, de las que 10 son de geografía de la población.

Fuente: Anales de Geografía de la Universidad Complutense de Madrid. núm. 1, 1981.

Universidad de Barcelona:

Período 1967-86: 47 Tesis doctorales, de las que 3 son de geografía de la población. 130 Memorias de Licenciatura, de las que 7 son de geografía de la población.

Fuente: Revista de Geografia. Barcelona, vol. XX, 1986.

Universidad de Valencia:

Período 1957-81: 20 Tesis doctorales, ninguna de geografía de la población.

Período 1957-79: 78 Memorias de Licenciatura, de las que 7 son de geografía de la población.

Fuente: Cuadernos de Geografía. Valencia, núms. 10 (1972), 15 (1974) y 25 (1979). 
Como se observa en el cuadro, es muy alto el porcentaje de los que declaran cultivar la Geografía de la población, un tercio de los «nuevos» geógrafos españoles, aunque como especialistas interesados sólo en geografía de la población son únicamente el $4 \%$ del total y el $12 \%$ de los que cultivan esta materia. Las últimas cifras pueden ser significativas sobre el futuro desarrollo de la geografía de la población, ya que aunque acusa su menor tradición respecto a la geomorfología o geografía agraria, debido a los problemas crecientes que afectan a nuestra población, así como por las características actuales de la participación del geógrafo en los estudios de planificación, es probable un mayor desarrollo de esta disciplina y de las investigaciones a ella dedicadas, de modo que la equiparación cuantitativa de nuestra dedicación a esta materia sea pareja al resto de los países más desarrollados. En este sentido, D. Noin estima que en Francia, los geógrafos que se interesan de manera única o predominante por la geografía de la población son el 6-7\% del total ${ }^{3}$.

El segundo indicador a que hacíamos referencia sobre el futuro interés de la geodemografía para los geógrafos españoles, es más específico del tema que nos ocupa; se trata de «la parcela concreta de información y/o análisis que ha cubierto el trabajo del geógrafo cuando participa en equipos de planificación». Según una reciente investigación del geógrafo profesional Juan Oliva Espallardo, donde presenta los resultados de una encuesta entre los geógrafos profesionales de España ${ }^{4}$, la «demografía» aparece como la materia más tratada por los geógrafos en los citados equipos, con un valor absoluto de 64 geógrafos -sobre un total de 77-, mientras el «medio físico» lo han tratado 54, la «infraestructura»50, «economía» 42, «sociología» 32, etcétera; en la frecuencia «siempre», la demografía aparece tratada por 20 geógrafos y el medio físico por 16, mientras en la frecuencia «muy a menudo», la demografía alcanza el valor 30 , muy por encima de la materia «sociología» con 19 , el «medio físico» e «infraestructura» con 18 cada una y la «economía» con 17.

En la publicación que comentamos se nos confirma que los geógrafos que participan en equipos de planificación lo hacen generalmente en funciones de información y asesoramiento, aunque cada vez acceden más a tareas de coordinación, codirección e incluso dirección. En cualquier caso, y ante el carácter pluridisciplinar de la planificación, Oliva Espallardo afirma que «debe defenderse la capacidad de cada profesión para intervenir en aquellas tareas que son más acordes con la preparación académica que cada uno ha recibido».

En este punto cabe preguntarse sobre la capacidad profesional geodemográfica que tan frecuentemente es solicitada a los geógrafos. Cuando en la encuesta que comentamos, se responde a la pregunta sobre la adecuación de los planes de estudio seguidos con el trabajo profesional que se solicita, la respuesta casi generalizada es negativa -70 sobre un total de 77 -. Las propuestas de los encuestados sobre materias que incorporarían a los planes de estudio -lógicamente con la finalidad de ejercer mejor como tales profesionales-, están orientadas a aquéllas que generalmente no están contempladas en los actuales planes de estudio, tales como información y tratamiento estadístico, ordenación del territorio, legislación urbanística o planeamiento urbano. Sin embargo, entre las materias que se proponen también las hay que ya están generalizadas en los planes de estudio vigentes, y en este sentido destacan la cartografía $\mathrm{y}$ fotointerpretación -18 propuestas- $\mathrm{y}$, en menor medida, la demografía -5 propuestas-. Esta última puede traducir la de aquellos geógrafos formados

3 NOIN, D., «La demogeografía en Francia», Est. Geográficos, 178-179. Madrid, 1985, p. 23.

4 OLIVA ESPALLARDO, J., «Geógrafos profesionales de la ordenación y planificación territorial (Censo General 1986)». Boletín de la Asociación de Geógrafos Españoles, núm. 5 (2. época), 1987, pp. 61-90. La encuesta recoge 77 respuestas sobre un total estimado de 250-300 geógrafos relacionados de algún modo con trabajos de planificación territorial. 
en Universidades donde no se imparte esta disciplina como específica -de 26 Universidades sólo figura como talen $19^{5}$-, o bien la insuficiencia del programa que han cursado para esta finalidad práctica.

La necesidad imperiosa de aumentar la formación e investigación demográficas también es manifiesta para otros científicos sociales que habitualmente se ocupan de esta temática. Así, entre los ejemplos más recientes, se puede citar el Editorial «Economía de la población», correspondiente al número 24 (1987) de la revista Economistas, del Colegio de Economistas de Madrid, donde se afirma «Los estudios de población en España no han ocupado hasta el presente, ni en la Universidad ni en la Administración, el campo institucional que en los países vecinos como Italia o Francia se les depara y que su relevancia social exigiría». Por su parte, la atención que los sociólogos prestan a los estudios de población es de sobra conocida, tanto en sus investigaciones como en la docencia universitaria, que, además preparan potenciar al más alto nivel con motivo de la actual reforma de los planes de estudio universitarios.

Los ejemplos aportados vienen a confluir, pues, en la insuficiente atención que hasta ahora se ha prestado en España al estudio de la población, al mismo tiempo que se es consciente de ello y de la necesidad de corregir esta situación. Para ello se busca aumentar tanto la cantidad y calidad de las investigaciones con finalidad práctica, como la formación a impartir para lograr una suficiente capacidad para la ejecución de tales investigaciones o, en su caso, para captar su necesidad y alcance.

\section{Los estudios de población: la necesidad de actualizar un útil con posibilidades múltiples según escalas y variables}

Los estudios de Geografía de la población son básicos para la planificación a distintas escalas, aunque llevarlos a nivel supranacional es más dificultoso y menos efectivo, ya que la planificación requiere un marco jurídico y económico que generalmente sólo se encuentra dentro de las fronteras nacionales o en otras siempre más reducidas, que pueden descender a sólo unas hectáreas de superficie, como es el caso de un barrio urbano. Por otra parte, los estudios de geografía de la población como apoyo para la planificación pueden enfocarse bajo las diversas variables geodemográficas, siempre dependiendo de la finalidad del estudio, lo que a su vez primará unas u otras interrelaciones. Así pues, el abanico de posibilidades es cuantioso, tanto por las escalas de planificación, como por las variables geodemográficas a utilizar y las interrelaciones a efectuar, así como por la necesaria renovación de los estudios a resultas de los frecuentes cambios que se operan en las tendencias demográficas, y las subsiguientes consecuencias sobre las políticas o el territorio.

En la actualidad la percepción del carácter aplicado de los estudios geodemográficos, es un tema que trasciende más allá de los especialistas, para convertirse en una preocupación de la que participan amplios sectores de la sociedad, aunque el uso de los estudios de población con finalidad práctica es muy antiguo. Así, entre otros ejemplos se pueden citar los mapas de densidades hechos por el inglés H. Harness en 1837 como estudio preparatorio para el trazado de los ferrocarriles, al objeto de conocer la distribución espacial de los habitantes,

5 Vid. Boletín de la Asociación de Geógrafos Españoles, n. 9 9, abril de 1983, pp. 4-5. También en Puyol Antolín, R., «La Geografía de la población a la búsqueda de sus señas de identidad. (Resultados de una encuesta sobre la enseñanza de la disciplina en las universidades españolas)». Est. Geográficos, 178-179, 1985, pp. 49-60. 
futuros clientes del ferrocarril ${ }^{6}$, o el ejemplo más general de los antiguos recuentos de población, anteriores a los censos modernos, entre cuyas finalidades esenciales estaba la recaudación de impuestos y las levas militares, a tal punto que alguno de ellos, como el Censo de Castilla de 1591 era conocido en su tiempo como el «Censo de los millones», pues se hizo para el cobro de un impuesto extraordinario de ocho millones de ducados ${ }^{7}$.

En las páginas que siguen exponemos algunas reflexiones sobre la utilidad práctica de los estudios geodemográficos, aunque limitadas sólo a algunas temáticas y escalas, pero que estimo trascendentes en la actualidad, al menos para amplias zonas de España; a este ámbito espacial hace también referencia el apartado final, dedicado a mostrar la deficiente cobertura temática a escala municipal, que caracteriza a las fuentes disponibles en la actualidad.

\section{2.a. Los estudios a escala nacional}

En la planificación a nivel nacional los estudios de población son hoy de gran trascendencia, debido a los rápidos e intensos cambios que experimenta la población, y que afectan a su distribución espacial, a la movilidad, fecundidad, a los modelos familiares, a la población económicamente activa, al alargamiento de la vida, a las causas de mortalidad, etcétera. Los cambios en muchas de estas variables, tomadas o consideradas como macrocifras, a nivel de Estado, tienen o tendrán en un futuro cercano consecuencias críticas para la economía, el bienestar o el equilibrio entre los territorios de la nación. Así, estos cambios pueden suponer problemas o readaptaciones importantes para el sistema educativo, sobre todo en sus niveles obligatorios, para el sistema de pensiones, para el sistema hospitalario, etcétera.

Conocer los efectivos demográficos actuales y sus comportamientos, es ipso facto prever la población y sus características demográficas en el futuro cercano, y por tanto sus implicaciones económico-sociales «positivas» $\mathrm{y}$ «negativas». En este sentido basta citar como muestra el descenso de los nacimientos en España, que entre 1976 y 1984, en ocho años, se han reducido casi en un tercio, al pasar de 677.456 a 465.709 nacimientos, con descenso que continúa aunque para los últimos años sólo conocemos algunas cifras regionales, como las de la Comunidad Valenciana, que entre 1981 y 1986, en 5 años, se han reducido casi en una cuarta parte, desde 54.669 a 42.431 nacimientos; el País Vasco, también entre 1981 y 1986 ha reducido su natalidad en un $28,6 \%$, situándose la tasa de la última fecha en 9,25 por $1.000^{8}$, mientras la natalista Murcia perdía un 13,7\% entre 1981 y 1985, con tasa de 14,4 por 1.000 en la segunda fecha'.

Las consecuencias de este brutal descenso de los nacimientos, a efectos nacionales o regionales pueden ser de variado signo según el plazo a que se contemplen. Así, durante un período muy inmediato tal descenso de nacimientos puede ser «positivo», ya que permitirá

6 NOIN, D., Géographie de la population. Paris, Masson, 1979, p. 34

7 MONTES FERNÁNDEZ, J., «Presentación» del Comentario al Censo de Población de las provincias y partidos de la Corona de Castilla en el siglo XVI. Edición facsímil realizada por el INE en 1982 de la publicación que de tal censo hizo D. Tomás González en 1829.

8 GALDÓS URRUTIA, R. y RUIZ URRESTARAZU, E., «Transformaciones en la dinámica natural de la población en el País Vasco (1970-86). De una alta natalidad a valores infraeuropeos». Análisis del desarrollo de la población española en el período 1970-1986, Madrid, Ed. Síntesis, 1989, pp. 229-235.

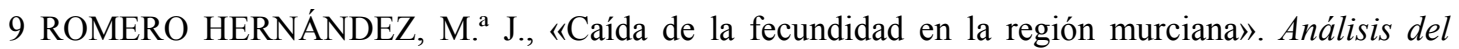
desarrollo de la población española en el período 1970-1986, Madrid, Ed. Síntesis, 1989, pp. 223-228. 
reducir los gastos generados por la infancia, e incluso mejorar la calidad de la enseñanza primaria sin aumentar los recursos destinados a ella, pues muchas escuelas reducirán el número de sus alumnos, aunque también se originarán problemas de ajuste espacial de profesorado. Unos años después, cuando estas generaciones menguadas lleguen a edad laboral, pueden conllevar aspectos «positivos», pues sin esfuerzos especiales se puede reducir el paro actualmente existente, pero los efectos económicos negativos se presentan simultáneamente y con gravedad, pues si disminuye el ingreso de los efectivos que trabajan, disminuye ipso facto la recaudación de impuestos o se habrá de aumentar la carga impositiva para compensar la disminución de cotizantes. No obstante, también existe otra vía fácil en principio para corregir este desajuste económico de los sistemas sociales, y es el recurso a la inmigración desde los cercanos países africanos, aunque, como es sabido, esta solución en Europa no se contempla con entusiasmo por las previsibles dificultades de integración social que pueda conllevar.

Si avanzamos hacia la cumbre de la pirámide de edades, el descenso de nacimientos provoca simultáneamente un envejecimiento de la sociedad, es decir aumenta la proporción -independientemente de las cifras absolutas- de los no activos por su edad, es decir de los de más de 65 años según la actual legislación laboral. Así el grupo de pensionistas se engrosa relativamente porque no son compensados en las cifras censales por la llegada de los nacimientos, $\mathrm{y}$, además, también aumentan absolutamente porque, si no cambian negativamente los actuales niveles de vida, sanitarios y los del medio ambiente, la vida media puede alargarse, no sólo con edades extremas -que es más difícil-, sino sobre todo por el número de personas que sobrepasan la edad de jubilación y por una permanencia más larga en situación de pensionistas.

El envejecimiento como proporción de personas con más de 65 años, aunque aumenta a partir del momento en que empieza la disminución de nacimientos, desde el punto de vista económico la situación todavía no es grave en el caso de España, porque aún han de entrar en la actividad laboral las abultadas generaciones que nacieron entre 1960 y 1975 . El envejecimiento español se agravará económicamente cuando las generaciones menguadas que están ahora en la base de la pirámide, lleguen a la edad laboral y a la edad de procreación, pues disminuirán los cotizantes y la base de la pirámide se reducirá por baja natalidad y por generaciones de padres muy menguadas. Es decir, el descenso prolongado de la fecundidad produce el envejecimiento de modo automático, siempre, claro está, que no intervenga una inmigración masiva, lo que no es probable a escala nacional, aunque sí a escala regional, provincial o municipal como resultado de movimientos migratorios interiores, tendentes a ajustar los desequilibrios económicos territoriales.

La utilización de los estudios de población para adecuar las políticas y las previsiones sociales a la nueva situación demográfica, es sin duda un aspecto muy sobresaliente de la utilidad práctica de esta disciplina, que debe ser divulgada y cultivada con los medios precisos, no siempre existentes en nuestro país. Por otra parte, los estudios demográficos también pueden informar sobre la conveniencia o no de ensayar nuevas políticas familiares tendentes en la actualidad a corregir positivamente la fecundidad, ya que según ponen de manifiesto numerosas investigaciones, la actual crisis de fecundidad parece estar motivada en una parte de la sociedad, por las dificultades económicas, aunque en muchas ocasiones estas dificultades son sólo relativas, pues están determinadas por umbrales de bienestar económico que se desea alcanzar, independientemente de si son más o menos altos.

En cualquier caso, parece que los factores que han provocado la caída de fecundidad, aunque en parte estén influidos por la economía, están sin duda más determinados por las nuevas actitudes culturales en su sentido más amplio y variado, que van desde la sexualidad al feminismo, pasando por los nuevos modelos y actitudes ante la familia, es decir cómo debe 
concebirse ésta -con vínculos jurídicos, en situación de cohabitación...-, o qué número de miembros debe tener ${ }^{10}$.

Pese a la escasez de estudios específicos tendentes a poner de manifiesto la vertiente práctica de las investigaciones sobre la población, creo que es significativo de las futuras preocupaciones por este tipo de investigaciones el reciente simposio organizado por el Ministerio de Economía y Hacienda con el título Tendencias demográficas y planificación económica, en el que una veintena de expertos nacionales y extranjeros han presentado excelentes estudios sobre el descenso de la fecundidad en España y en los países industrializados, sobre sus causas, sus repercusiones y efectos, fundamentalmente los de tipo económico, sanitario, educativo, mercado de trabajo, sistema de pensiones y las repercusiones sobre el urbanismo y por tanto en la política territorial, cerrando las intervenciones un capítulo dedicado a las políticas de población, en el que intervinieron responsables políticos españoles en la materia, que no parecían especialmente inquietos por la situación española, a pesar de haber llegado a un umbral de notable deterioro en la fecundidad, más grave que el que registran muchos países europeos que sí han emprendido políticas familiares activas, pese a su elevado costo financiero; la justificación de estas inversiones las contemplan, evidentemente, a medio plazo, ya que en los actuales niveles una ligera subida de la fecundidad puede suponer o no la garantía del reemplazo de generaciones. En este sentido la actitud de los últimos gobiernos franceses es el caso más representativo, entre los países occidentales, sobre inversiones en políticas familiares activas, consiguiendo mantener tasas estables en la última década: natalidad de 13,6 por 1.000 en 1976 y de 13,9 en 1985, mientras en España esta tasa disminuyó un 36\% entre 1976 y 1984, situándose en la última fecha en 12,1 por 1.000. En la Comunidad Valenciana el descenso de la natalidad entre 1976 y 1986 ha sido del $42 \%$, registrándose en la última fecha entre 11,7 por 1.000 en Alicante y 10,0 por 1.000 en Castellón ${ }^{11}$.

\section{2.b. La geografía de la población en la planificación rural}

Se exponen a continuación algunas reflexiones o directrices sobre aspectos prácticos o aplicables de la geografía de la población en ámbitos rurales. La población rural es, sin duda, una de las subpoblaciones europeas, y en concreto española, cuya evolución reciente experimenta cambios sustanciales, generalmente en el sentido de su degradación demográfica, pero también en sentido positivo en espacios generalmente reducidos. En cualquiera de los casos, es claro que los estudios de geodemografía pueden ser especialmente útiles en la planificación y ordenación de los diferentes espacios rurales.

En síntesis, la población rural ha evolucionado durante el último cuarto de siglo, en el sentido de acelerar el éxodo que ya se producía desde el siglo XIX, lo que importa tener en cuenta para así entender mejor la actual degradación demográfica, irreversible en muchos

10 GOZÁLVEZ PÉREZ, V., «Dinámica natural de la población española, 1970-1986». Análisis del desarrollo de la población española en el periodo 1970-1986, Madrid, Ed. Síntesis, 1989, pp. 191-207.

11 GOZÁLVEZ PÉREZ, V., «La población Valenciana en 1986: la evidencia de una fuerte y abierta crisis». Cuad. de Geografía, núm. 42. Valencia, 1987. 
casos si se abandona a su actual dinámica, esto es, si no se introducen medidas correctoras mientras haya posibilidad.

Durante el último siglo, la despoblación rural se ha producido con distintas intensidades en diferentes períodos, motivadas en unos casos por las crisis agrícolas -generalmente antes de 1950-, o por el desarrollo y atractivo urbanos en las últimas décadas. En cualquier caso la emigración de rurales siempre es selectiva, pues afecta con gran preferencia a los adultos jóvenes, y en el último cuarto de siglo más a las mujeres que a los varones. Las consecuencias negativas que la intensificación de este proceso tienen para la demografía y medio agrario son evidentes. Los nacimientos descienden en exceso, no sólo por la salida de los adultos jóvenes, sino también por un acusado aumento de la soltería, pues se ha alterado muy sustancialmente la relación entre sexos, sobre todo en los municipios con menor población, y especialmente en aquellos cuya capital municipal tiene censo exiguo, debido a que la falta de población concentrada conlleva carencia de servicios hoy considerados imprescindibles, lo que incentiva el éxodo rural.

El resultado es que la mayoría de los municipios rurales, aún en el supuesto improbable que vieran paralizada su emigración, continuarían en despoblación absoluta porque en ellos mueren más habitantes de los que nacen, y por tanto la despoblación absoluta de muchas de estas áreas puede ser sólo cuestión de pocas décadas. Mientras esta despoblación absoluta no haya llegado, los mecanismos correctores pueden introducirse creando servicios y empleos alternativos, capaces no sólo de retener a los jóvenes que aún quedan, sino también concentrando en los núcleos rurales la población más desasistida del diseminado.

Las medidas económicas tendentes a evitar la despoblación rural excesiva, como muy costosas al erario público, deben apoyarse también en serios y finos estudios de su población. Naturalmente, cuando se habla de medidas de planificación para fijar la población, o al menos cierto umbral de población, en las áreas rurales, no se piensa en solventar con ello problemas de abastecimiento agrario, lo que se consigue mejorando la productividad con el avance tecnológico, sino que se trata de evitar con ello problemas sociales y ecológicos que se derivan de esta despoblación, y que afectan tanto a las áreas que se quedan abandonadas como a las que reciben más población de la que prevén sus posibilidades.

Es evidente que el éxodo agrícola es necesario con el desarrollo de la economía, desde la agraria a la industrial y terciaria. Lo que no es admisible, y es donde debe intervenir la planificación correctora de este proceso, es un éxodo rural que conlleve degradación demográfica a tal punto que suponga entrada en la dinámica del despoblamiento mecánico o automático.

De acuerdo con lo indicado, son útiles para la planificación rural, el análisis y valoración de las tendencias de los efectivos totales y de su reparto espacial, la dinámica de su movimiento natural, de los movimientos migratorios, así como de la estructura por edades y sexos, y todas estas variables consideradas sobre el espacio, en mapas, e interrelacionadas.

Entre todas estas variables, actualmente una de las que requieren estudios más urgentes es el envejecimiento rural, ya muy avanzado, pues su conocimiento resulta de gran utilidad para la planificación demográfica correctiva, debido a las nefastas consecuencias demográficas y económicas en los territorios donde el envejecimiento se acentúa.

Como cualquier otro hecho geodemográfico, la cartografía del envejecimiento rural a escala municipal, se revela como un utilísimo instrumento para la planificación, ya que como muestran los estudios realizados, la intensificación del envejecimiento actúa en mancha de 
aceite, con sus tasas más altas en el centro del área originaria, que engloba progresivamente a los municipios contiguos. Es decir, el envejecimiento rural no es un hecho demográfico que se presenta aislado; además su intensificación aparece superpuesta al menor tamaño de los núcleos rurales, es decir a menor tamaño, mayor envejecimiento, hecho que también se corrobora en las pirámides de edades de las distintas categorías de municipios según su volumen poblacional. Por consiguiente, las medidas correctoras y preventivas de la degradación demográfica por envejecimiento, tienen un útil excelente en estos mapas, pues permiten detectar las áreas que en períodos futuros pueden fácilmente evolucionar hacia situaciones más deterioradas $\mathrm{y}$, por tanto, se pueden emprender acciones correctoras. La tipificación demográfica de los municipios rurales puede resultar, pues, un excelente medio para la planificación en este ámbito.

Otra variable demográfica cuyo conocimiento es de gran trascendencia en la tan necesaria planificación de las áreas rurales, es el de su potencial en mano de obra. Ante el generalizado envejecimiento de esta sociedad, hay que prever el montante de su población activa, así como sus características en edad y sexo, todo ello justificado ante la rentabilidad de las inversiones públicas o privadas que aquí se pueden hacer. Habría que valorar la mano de obra disponible en un momento dado, y sobre todo su previsión a corto plazo, especialmente si se trata de zonas en decadencia demográfica (envejecimiento, despoblación), y en este sentido también habría que valorar la mano de obra agrícola liberable atendiendo a la evolución o características de la agricultura y del resto de actividades en esa zona, así como por el tan frecuente subempleo agrario.

\section{2.c. Los estudios geodemográficos en la planificación de los espacios urbanos}

A efectos de planificación urbana, los estudios de geodemografía cabría realizarlos a diversas escalas de actuación: a nivel de áreas metropolitanas o urbanas, a nivel de ciudad o para espacios más reducidos, como barrios o sectores urbanos.

A escala de ciudad o de conjunto urbano un primer problema al que deben de atender adecuadamente los estudios geodemográficos es el de la dinámica de las cifras de población absoluta, es decir si hay o se prevee aumento, aumento muy acelerado o disminución de efectivos, pues las previsiones y posibilidades de la planificación deben ser diferentes en una ciudad o conjunto urbano que esté en expansión o en regresión demográfica. Es decir, es obvia la trascendencia que tiene planificar para el crecimiento demográfico o para un censo en disminución, situaciones extremas que, por otra parte, pueden y están presentes en la misma ciudad en fechas no distanciadas, como ocurre en las principales ciudades valencianas (vid. cuadro de la pág. siguiente).

Las tendencias del censo de una ciudad están sujetas o dependientes de variados factores, a veces con cambios más o menos bruscos en su trayectoria; el movimiento natural de la población y los movimientos migratorios son los que deciden la evolución censal, mientras su comportamiento está sujeto o depende de causas muy variadas y complejas. Las previsiones sobre los movimientos migratorios, tan decisivos en los censos urbanos, pueden ser especialmente difíciles y ofrecer tipologías muy variables, ya que aparte de la coyuntura económica general, cada ciudad evoluciona según sus características económicas específicas, que mueven a un determinado volumen de activos y a sus familias. Pero además, las 


\begin{tabular}{|c|c|c|c|c|c|c|}
\hline & \multicolumn{3}{|c|}{$\begin{array}{l}\text { Variación censal anual } \\
\text { en \% }\end{array}$} & \multicolumn{2}{|c|}{ Habitantes de hecho } & \multirow[t]{2}{*}{$\begin{array}{l}\text { Variación } \\
\text { en \% }\end{array}$} \\
\hline & $1961-70$ & 1976-81 & $1981-86$ & 1960 & 1986 & \\
\hline Valencia & 2,9 & 1,0 & $-0,3$ & 505.066 & 738.575 & 46 \\
\hline Alicante & 5,2 & 2,8 & 1,1 & 121.527 & 265.543 & 118 \\
\hline Elche & 6,7 & 2,0 & 1,3 & 73.320 & 173.392 & 136 \\
\hline Castellón & 5,1 & 3,0 & 0,5 & 62.493 & 129.813 & 108 \\
\hline
\end{tabular}

migraciones también pueden afectar de forma sustancial a subpoblaciones urbanas ajenas a la actividad económica (jubilados), que pueden seguir tendencias migratorias divergentes a las que siguen los activos y sus familiares; es decir los jubilados pueden emigrar o no acceder en notables contingentes de/hacia las ciudades de fuerte vocación económica, mientras son atraídos hacia áreas rurales, urbanas o núcleos residenciales o de vacaciones. Por todo ello, las previsiones sobre la evolución de cada población urbana concreta, requieren un conocimiento preciso de las distintas situaciones y potencialidades locales; a su vez la complejidad de las previsiones aumenta cuando se realizan para la planificación en áreas urbanas o metropolitanas, compuestas por núcleos más o menos numerosos, con funciones y posibilidades diferentes, pero que a su vez evolucionan de modo coordinado y dependiente del conjunto urbano en el que están integrados y del que económicamente forman parte.

Por otra parte, la necesidad de revisión de las planificaciones urbanas se acentúa con los cambios en las tendencias demográficas, ya que en caso de evolución negativa respecto a las previsiones, pueden afectar muy desfavorablemente a los ingresos de la hacienda municipal, mientras que la cantidad y calidad de servicios previsiblemente no puedan rebajarse en la misma proporción que la disminución censal.

Un hecho que puede adquirir gran importancia en la planificación urbana de determinados casos, son las proporciones o relaciones entre población residente y población transeúnte, pues el volumen de la última puede alterar sustancialmente las demandas de servicios en relación a la población residente (comercio, transporte, sanidad, etcétera).

El barrio es una escala de análisis geodemográfico privilegiada, pues es absolutamente representativo de las diferenciaciones sociales en el interior de la ciudad; estas diferencias son perfectamente legibles en cualquiera de las variables geodemográficas, tales como la actividad, la geografía electoral, el nivel de instrucción, la geografía escolar, el origen geográfico de la población, la estructura por edad y sexo, etcétera.

Los distintos barrios de una ciudad, de acuerdo a su evolución urbana o a su concepción inicial, albergan a poblaciones o vecinos con características sociodemográficas diferentes, que con frecuencia necesitan o requieren tratamientos urbanos específicos para gozar de una mayor confortabilidad urbana. Para que esto se pueda planificar o realizar es preciso conocer el montante, las características y estructuras de la población y subpoblaciones que allí viven, o de la que pretendemos que allí resida en el caso de rehabilitaciones urbanas, que tanta importancia adquieren en la actualidad. 
El conocimiento de la estructura por edades y sexos de la población de un barrio es, entre otras, una variable de conocimiento inexcusable para planificar servicios muy diversos, entre ellos los centros de enseñanzas o en su caso las necesidades de transporte escolar. Sin embargo el perfil general de la pirámide es insuficiente, también se requiere conocer el status social de esa población, sobre todo si se trata de enseñanza no obligatoria; casos extremos en este sentido pueden ser los barrios envejecidos de las áreas centrales y los barrios edificados recientemente ex novo y sin continuidad ${ }^{12}$; es decir, al mismo tiempo que se requiere conocer el perfil general de la pirámide, interesa no menos -al menos para ciertos casos- el de las subpoblaciones en ella integradas.

Las áreas metropolitanas o regiones urbanas son escalas de la urbanización actual que requieren una adecuada planificación; es obvio que los indicadores demográficos son esenciales para delimitar y planificar tales espacios, aunque para elaborar muchos de estos indicadores en nuestro caso no existe información con suficiente detalle, ni en variables demográficas ni en unidades espaciales, tal como se indicará después.

\section{Indicaciones sobre las fuentes geodemográficas}

Las fuentes geodemográficas españolas atienden a escalas privilegiadas como son los totales nacionales y los provinciales, a las que hay que añadir otros espacios menores como los municipios de las capitales provinciales, otros municipios a partir de un umbral más o menos alto de población, o conjuntos provinciales de población rural, urbana e intermedia. Sin embargo, tal como hemos indicado, a efectos de planificación resultan actualmente imprescindibles fuentes a nivel de todos los municipios, e incluso para espacios menores, fundamentalmente en las grandes ciudades. Asimismo se observa un notable retraso en incorporar a las fuentes oficiales publicadas datos sobre aspectos que, con toda evidencia, se requieren en la planificación actual, y además de forma periódica. Así, entre otros ejemplos elementales hay que citar los datos sobre movilidad habitual, como los que recogía por primera vez en España el Censo de población de 1981, pero que no se han llegado a publicar $^{13}$. Otro ejemplo no menos llamativo sobre las deficiencias de la información demográfica, es la carencia de datos a nivel de todos los municipios sobre aspectos tan necesarios y elementales como la distribución por edad y sexo o cifras absolutas de empleo en las distintas ramas de actividad; la información sobre el número de nacimientos y defunciones a escala municipal sólo se ha iniciado su publicación por primera vez en España en 1975.

Las repercusiones o utilidad de estas informaciones es evidente para la planificación del transporte, de las necesidades escolares, de los diversos aspectos que hemos señalado para el caso de la población rural -envejecimiento, mano de obra disponible, etcétera-, para la

12 Vid. en este sentido Crespo Giner, J., «La estructura por edad, sexo y estado civil» ap. Atlas sociodemográfico de la ciudad de Alicante. Alicante. Instituto de Estudios Juan Gil-Albert y Ayuntamiento de Alicante, 1987, 143 pp.

13 La información solicitada en las hojas censales era: 1) lugar de trabajo o estudio, desglosados en a) no tiene lugar fijo de trabajo, b) trabaja o estudia en el municipio de residencia, y c) trabaja o estudia en otro municipio. 2) Desplazamiento al lugar de trabajo o estudio, y dentro de esta propuesta, a) medio de transporte utilizado, con nueve posibilidades de respuesta, $\mathrm{y}$ b) tiempo empleado (en minutos) en el trayecto de ida, con cinco umbrales en la respuesta. 
delimitación de las áreas metropolitanas o para detectar la expansión de la urbanización rural, entre otros ejemplos de planificación y previsión elementales en la actualidad. Así, según estas indicaciones, se concluye que ciertas previsiones a escala municipal o comarcal pueden hacerse con carencia de datos básicos, o, lo que es más desalentador, no se siente la necesidad de fundamentar con esta información la planificación y previsiones a escala local.

Aunque el estudio geodemográfico de los barrios es una necesidad obvia y absolutamente compartida a efectos de planificación urbana, también es un hecho igualmente universal, en el caso de España, el absoluto inconfort estadístico para este tipo de estudios geodemográficos. Las investigaciones a esta escala y con finalidad práctica, requieren información específica que en absoluto, o nunca, es recogida en las estadísticas disponibles, ya muy deficitarias incluso a escalas más amplias, tal como se ha indicado. A escala intraurbana el geógrafo o científico social de la población, si desea realizar un trabajo correcto tendrá que hacer personalmente una costosa elaboración de la fuente, y se verá impotente para estudios de mayor cobertura espacial.

Creo que puede ser positivo señalar estas carencias estadísticas, sobre todo justificando la necesidad de su uso, aunque no podamos esperar una solución total e inmediata a nuestras demandas. Debemos divulgar o hacer ver la utilidad práctica de los estudios geodemográficos, pues el beneficio es común y, además, creo que nuestros enfoques espaciales, en este campo, con una cartografía abundante, adecuada y bien ejecutada, cuando son conocidos sin duda son bien recibidos. 